\title{
Platine d'expérimentation MicroMAG : à la découverte de la machine synchrone autopilotée
}

\author{
B. NOGAREDE, D. HARRIBEY, Y. LEFEVRE, F. PIGACHE \\ Bertrand.nogarede@laplace.univ-tlse.fr \\ INPT/ENSEEIHT, 2 rue Camichel, 31071 Toulouse cedex 07
}

RESUME : L'objectif de cet article est de présenter la platine d'expérimentation MicroMAG utilisée en $1^{\text {ère }}$ année d'école d'Ingénieurs ENSEEIHT (niveau L2). Cette maquette permet d'appréhender les principes de fonctionnement d'une machine synchrone autopilotée, à partir d'une approche pratique des phénomènes physiques mis en jeu. Cet article détaille tout d'abord la maquette de travail puis décrit les différentes étapes de l'étude en s'appuyant sur les bases théoriques de l'électrodynamique.

Mots clés : électrodynamique, machine synchrone autopilotée, bobinage de machine triphasée, entrefer axial, forces de Laplace, stratégie d'alimentation, commutations.

\section{INTRODUCTION}

Lors de la première année de formation des élèves ingénieurs au département Génie Electrique de l'ENSEEIHT, les étudiants abordent les principes des machines électromagnétiques sous un aspect tout d'abord théorique [1], ensuite confortés par des travaux pratiques pour l'assimilation des lois élémentaires mises en jeu. La maquette MicroMAG, élaborée par le groupe de recherche en Electrodynamique (GREM3LAPLACE) pour le département Génie Electrique Automatique de l'INP/ENSEEIHT, a pour objectif d'illustrer de manière concrète et originale ces lois et phénomènes : les étudiants sont amenés à se poser les questions fondamentales qui conduisent au dimensionnement, à la conception et à la caractérisation de leur propre machine.

L'objectif de cet article est de présenter la platine développée ainsi que le déroulement des expérimentations, appuyé par l'étude analytique et sa validation. Tout d'abord, la structure modulaire de la maquette permettant la constitution, par l'étudiant luimême, de la machine synchrone autopilotée est détaillée. Une seconde partie s'attache à décrire l'interaction électromagnétique utilisée, mettant ainsi en évidence les forces de Laplace. Le déroulement de la séance est ensuite exposé d'un point de vue pédagogique, depuis la modélisation de la structure et son dimensionnement partiel jusqu'à l'étude expérimentale des différentes stratégies d'alimentation employées pour la création du champ tournant. Les performances du convertisseur électromécanique ainsi constitué sont alors globalement testées et caractérisées.

\section{PRESENTATION DE LA MAQUETTE}

La platine d'expérimentation MicroMAG, est destinée à découvrir, connaître et quantifier les principes élémentaires d'interaction champ/courant utilisés dans les convertisseurs électromécaniques d'énergie. A cette fin, l'approche pédagogique retenue consiste à proposer à l'étudiant la construction progressive d'une machine synchrone autopilotée. La réalisation pas à pas et didactique de cet ensemble doit amener l'utilisateur à se poser les bonnes questions quant aux différentes fonctions qui constituent le procédé d'autopilotage couramment exploité dans les machines à courant continu et les machines à commutation électronique.

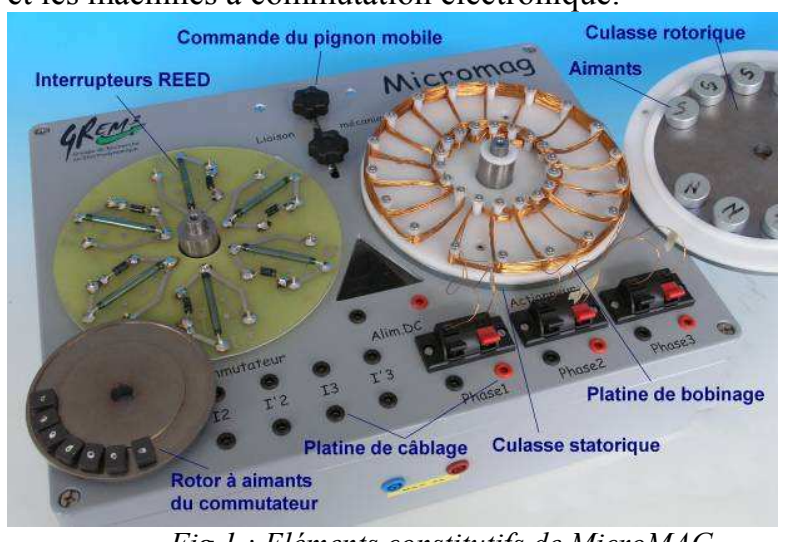

Fig 1 : Eléments constitutifs de MicroMAG

La plateforme MicroMAG est composée d'une partie moteur, d'une partie commutateur et de divers éléments désignés sur la figure 1 :

- Le moteur étudié est un moteur à entrefer axial. Il est composé d'un palier central solidaire du boîtier destiné à guider en rotation un disque métallique qui sert de culasse rotorique et sur lequel sont disposés les aimants Néodyme-fer-bore. Une série de perçages sont aménagés sur le dessus du disque rotorique pour pouvoir y accrocher un dynamomètre afin de procéder à des mesures de couple statique. Une platine amovible isolante sert de support au bobinage et enfin une culasse statorique permet au flux magnétique de se refermer.

- La partie commutateur est équipée d'un palier identique guidant le disque rotorique équipé de ses aimants ferrite. La culasse statorique est coiffée par un circuit imprimé amovible sur lequel sont soudés des interrupteurs magnétiquement commandés (Relais REED). Les ouvertures et fermetures de ces interrupteurs sont pilotées par les aimants rotoriques du commutateur d'une fréquence maximale de l'ordre de la centaine de Hertz. Des diodes de roue libre sont placées en parallèle sur 
ces interrupteurs afin de limiter les arcs électriques lors de leur ouverture et ainsi préserver les contacts.

- Le boîtier est équipé d'un troisième palier mobile monté sur glissières qui permet de lier mécaniquement le moteur au commutateur. Chacun des paliers étant équipé d'une roue dentée, la liaison mécanique (Fig 2) est assurée dès que le pignon mobile est amené au contact des deux autres. Des vis moletées assurent le maintien du pignon mobile en position embrayée ou débrayée.

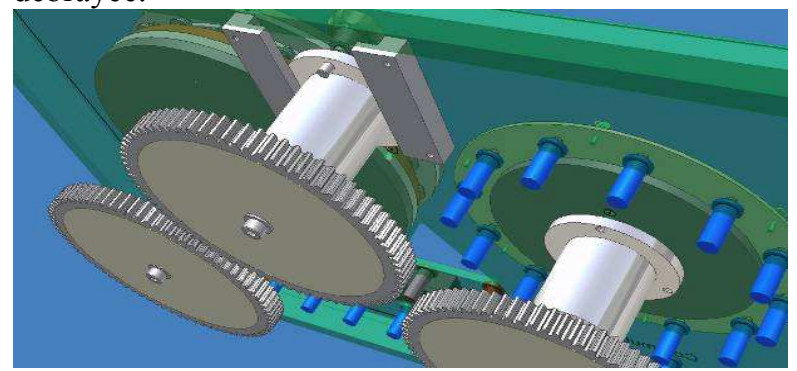

Fig 2 : Pignons de liaison

Le passage du fonctionnement en boucle ouverte au mode « autopiloté » est ainsi simplement réalisé.

- Enfin, les prises de contact des phases moteur, de l'alimentation DC ainsi que des interrupteurs sont ramenés sur des connecteurs de type banane situées sur le boîtier permettant ainsi de réaliser localement le câblage de l'ensemble machine-commutateur (Fig 3).

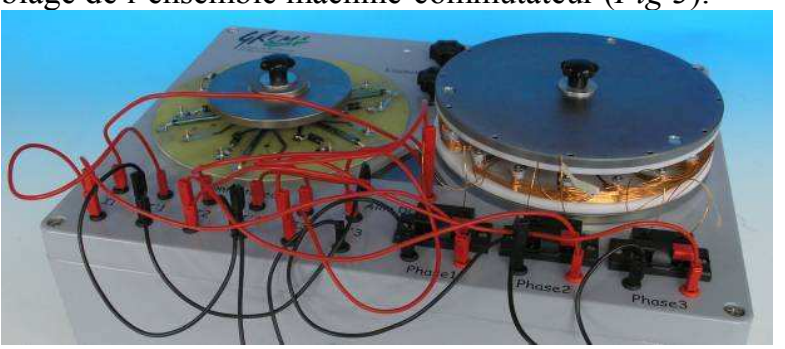

Fig 3 : MicroMAG prêt à fonctionner

MicroMAG offre la possibilité de réaliser plusieurs configurations de machine et d'autopilotage. Par contre, la liaison mécanique directe et de rapport de réduction unitaire entre les arbres moteur et commutateur ne permet d'envisager qu'une machine bipolaire.

La configuration actuelle de MicroMAG est plutôt destinée à étudier une structure triphasée de moteur autopiloté, mais pourrait, en disposant différemment les relais REED, d'envisager des structures monophasées ou diphasées.

L'arc polaire des aimants rotor de l'actionneur et du commutateur est ajustable et permet d'envisager les commandes classiques en créneaux de $120^{\circ}$ ou $180^{\circ}$. La platine de bobinage quant à elle peut au maximum accueillir un bobinage triphasé à trois conducteurs par phase. Là encore, moyennant d'aménager différemment la configuration de ces platines, il est facilement envisageable de réaliser des bobinages plus complexes (à pas raccourci, imbriqués...). L'alimentation continue nécessaire au fonctionnement du moteur doit pouvoir fournir 2,5 Ampères sous $15 \mathrm{~V}$.

\section{MODELISATION}

\subsection{Interactions Champ-Courant}

La machine étudiée étant constituée par une structure à entrefer axial sans encoches, le modèle utilisé pour étudier le moteur est basé sur la loi de Laplace et la loi de l'induction électromagnétique.

Sous l'hypothèse simplificatrice d'un champ magnétique inducteur $\mathrm{B}$ à distribution uniforme, la force mécanique $\mathrm{F}$ développée sur un conducteur de longueur 1 parcouru par un courant I est donnée par (fig 4) :

$$
\mathrm{F}=\mathrm{B} \text { I } 1
$$

- Sous cette même hypothèse d'uniformité du champ, la force électromotrice induite E sous l'effet du mouvement du conducteur animé d'une vitesse $\mathrm{V}$ perpendiculairement au champ inducteur s'écrit (Fig. 5):

$$
\mathrm{E}=\mathrm{B} \mathrm{V} 1
$$

\subsection{Calcul du champ magnétique}

Avant de réaliser le bobinage, les étudiants calculent le nombre de spires nécessaires pour obtenir un couple nominal donné (par exemple 0.15 N.m).

Pour faire ce calcul, il faut estimer la distribution et l'intensité du champ magnétique le long d'un conducteur. Ce calcul préalable peut-être réalisé par simulation numérique ou à l'aide d'un calcul analytique.

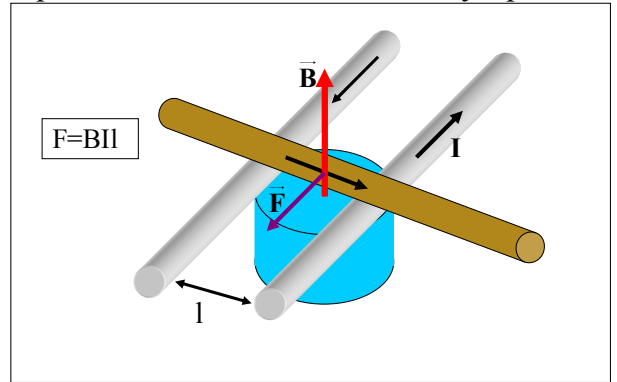

Fig. 4 : Force exercée sur un conducteur plongé dans un champ magnétique uniforme et parcouru par un courant

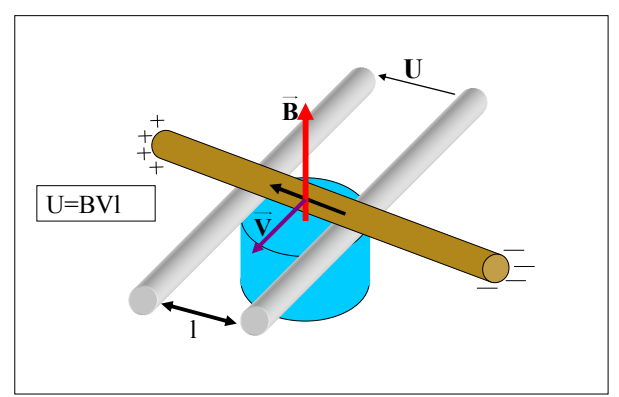

Fig. 5: F.e.m induite U aux bornes d' un conducteur en mouvement dans un champ magnétique uniforme

D'un point de vue pédagogique, il est préférable d'utiliser un modèle analytique. Ceci constitue une bonne occasion d'utiliser concrètement les résultats des cours théoriques. L'étudiant est également amené à bien comprendre par lui-même les phénomènes mis en jeu et à formuler des hypothèses simplificatrices appropriées. La figure 6 montre une vue en 3D de la culasse supérieure avec les aimants et de la culasse inférieure. 


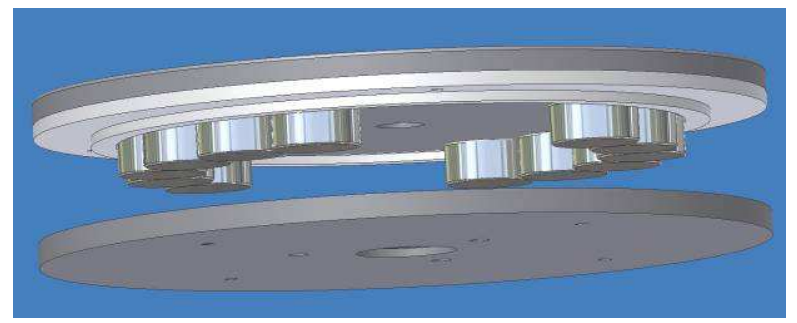

Fig. 6 : Vue en 3D des parties magnétiques du moteur

Malgré la forte valeur de l'entrefer, on peut supposer, dans le cadre d'une étude qualitative, que le champ est parallèle à l'axe de rotation et que les perméabilités des deux culasses sont infinies. Ensuite, en utilisant le théorème d'Ampère, la conservation du flux et les relations constitutives des différents milieux magnétiques, on peut calculer le champ magnétique au niveau des conducteurs. La figure 7 montre le contour orienté $\Gamma$ le long duquel est appliqué le théorème d'Ampère.

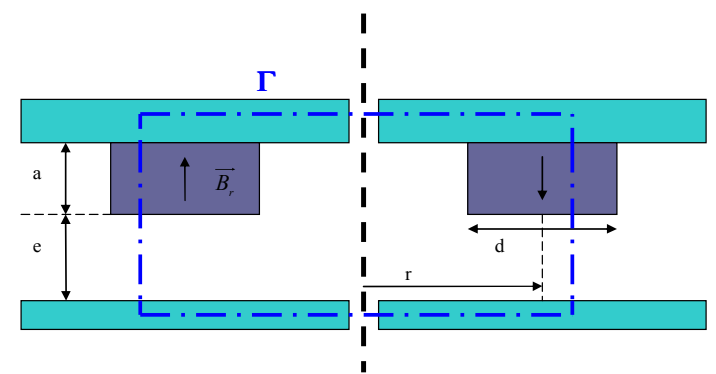

Fig. 7 : Contour Гretenu pour le théorème d'Ampère En négligeant la circulation du champ d'excitation magnétique dans le fer, l'expression de l'intensité B du champ magnétique au niveau des conducteurs est déduite:

$$
\left.\mathrm{B}=\frac{J}{\left(\mathrm{a}+\mu_{\mathrm{ra}} \mathrm{e}\right.}\right)
$$

où $J$ est la polarisation de l'aimant, $\mu_{\mathrm{ra}}$ la perméabilité relative de l'aimant, a l'épaisseur des aimants et e celle de l'entrefer (Fig. 7).

\subsection{Couple et f.e.m. pour une spire}

Enfin à partir des expressions (1) et (2), on déduit l'expression de la f.e.m dE et du moment du couple $\mathrm{dC}$ sur un élément du conducteur de longueur dr se trouvant à une distance $\mathrm{r}$ de l'axe :

$$
\begin{aligned}
& d E=B \omega r d r \\
& d C=B I r d r
\end{aligned}
$$

Dans ces expressions, I est le courant d'alimentation qui est ici généré par une alimentation continue stabilisée et $\omega$ la vitesse de rotation. Le moment du couple et la f.e.m relatifs à un conducteur sont obtenus en intégrant ces deux expressions le long du conducteur (Fig. 8 ). Une spire ayant deux conducteurs situés sous deux pôles de sens opposés, le couple Cs maximal et la f.e.m maximale Es pour une spire sont:

$$
\begin{aligned}
& C_{s}=B I\left(R_{2}^{2}-R_{1}^{2}\right) \\
& E_{s}=B \omega\left(R_{2}^{2}-R_{1}^{2}\right)
\end{aligned}
$$

$\mathrm{R} 1$ et $\mathrm{R} 2$ étant respectivement le rayon interne et externe de l'aimant (Fig. 8),

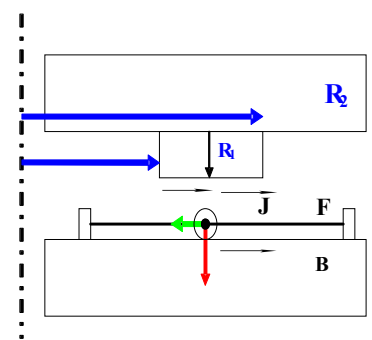

Fig. 8 : Force exerçant sur un élément de conducteur

En divisant le couple nominal par le couple unitaire Cs, on détermine le nombre de spires.

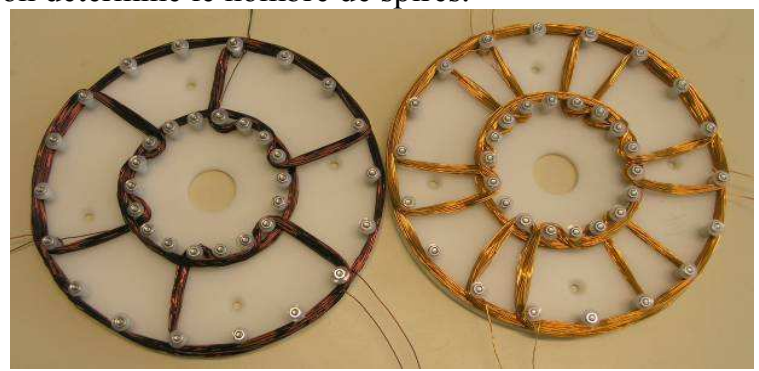

Fig. 9 : Photographie de deux bobinages triphasés à une et deux encoches par pôle et par phase réalisé par les étudiants

Les étudiants peuvent ensuite réaliser le bobinage. Pour un bobinage à pas diamétral, compte tenu du nombre total de plots, ils peuvent réaliser un bobinage à 1,2 ou 3 encoches par pôles et par phase (Fig. 1 et 9). On appelle ici 'encoche', par analogie à un moteur classique, l'espace entre deux plots (Fig. 9).

\section{STRATEGIE D'ALIMENTATION}

On appelle arc polaire la largeur angulaire d'une couronne d'aimants sous un pôle.

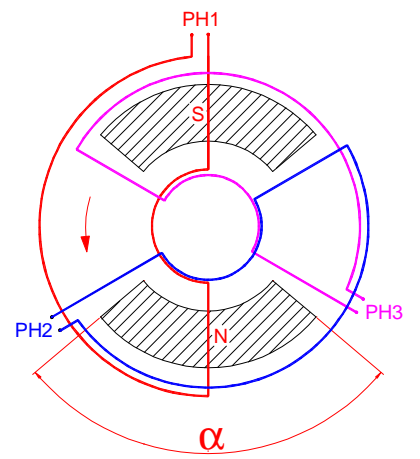

Fig. 10 : Position initiale du rotor par rapport aux phases du moteur

La figure 10 montre, dans le cas d'un bobinage à une encoche par pôle et par phase, une configuration particulière des couronnes d'aimants par rapport aux trois phases du stator. Dans le cas présent, l'arc polaire $\alpha$ vaut $100^{\circ}$. On prendra cette configuration comme posi- 
tion initiale des aimants que l'on fait tourner dans le sens trigonométrique (anti-horaire).

\subsection{Couple statique « par phase »}

Pour élaborer la stratégie d'alimentation on introduit la notion de couple statique. Ainsi, par exemple, le couple statique associé à la phase 1 , résulte de l'alimentation de cette phase par un courant continu d'intensité I. Si le rotor est entraîné par un moyen extérieur en maintenant le courant I constant, on obtient la courbe de couple statique de la phase 1 tracée en fonction de la position du rotor (Fig 11).

On peut obtenir le couple statique de la phase 1 en raisonnant à partir de la figure 10. Dans la position initiale, le couple est égal à la valeur maximale théorique Cs. Quand on fait tourner le rotor dans le sens trigonométrique, le couple se maintient à cette valeur tant que l'aimant ne découvre pas le conducteur (distance angulaire parcourue inférieure à $\alpha / 2$ ). Le couple s'annule ensuite lorsque les conducteurs de la phase sont situés dans la zone interpolaire.

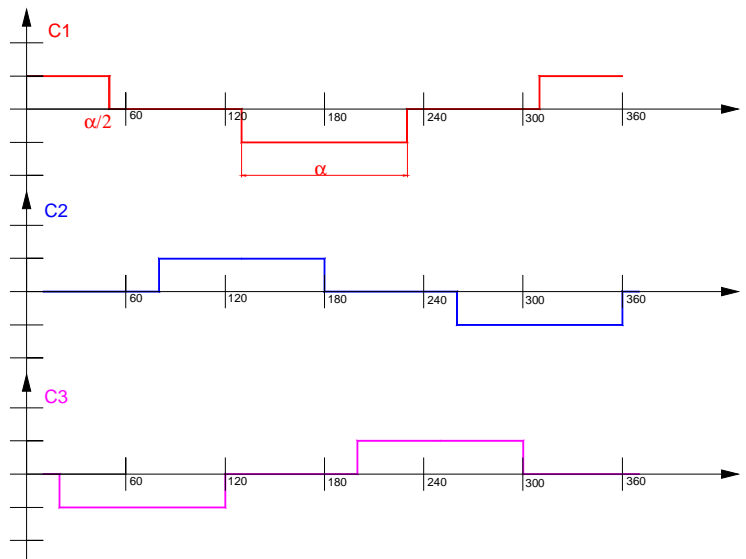

Fig. 11 : Couples statiques des trois phases en fonction de la position du rotor - La position initiale est montrée sur la figure 10

Lorsque le rotor aura parcouru un angle de $\pi-\alpha / 2$, le champ magnétique au niveau des conducteurs de la phase s'inversera de même que le moment du couple qui se maintient à la valeur -Cs jusqu'à la prochaine annulation du champ magnétique, soit jusqu'à la position $\pi+\alpha / 2$.

Le couple va ensuite s'annuler et retrouver sa valeur maximale $\mathrm{Cs}$ à la position $2 \pi-\alpha / 2$. Il se maintient à cette valeur jusqu'à la position initiale.

Les couples statiques des trois phases sont présentés sur la figure 11. Les courbes relatives à la phase 2 et 3 se déduisent respectivement de celle de la phase 1 par simple décalage de $2 \pi / 3$ et $4 \pi / 3$.

Compte-tenu de l'expression du couple et de la f.e.m (4), pour une positon donné on a, dans le cadre des hypothèses initialement formulées :

$$
C_{s} \omega=E_{s} I
$$

Ainsi, pour avoir une image du couple statique associé à une phase, il suffira de mesurer sa f.e.m. à vide.

\subsection{Couple statique « entre deux phases »}

Lorsqu'on connecte les phases en étoile, l'alimentation des phases du moteur avec un courant continu conduit à constituer un circuit pouvant être formé :

- de deux phases montées en anti-série,

- $\quad$ soit d'une phase en anti-série avec les deux autres connectées en parallèle.

Lorsque les phases 1 et 2, par exemple, sont alimentées en anti-série par un courant continu, pour une position donnée du rotor, le moment du couple résultant s'écrit :

$$
\mathbf{C}_{12}=\mathbf{C}_{1}-\mathbf{C}_{2}(7)
$$

A partir des courbes de couple statique des trois phases (Fig. 11), il est aisé de déduire la courbe de couple statique « entre deux phases » associée à l'alimentation simultanée de deux phases (Fig. 12).

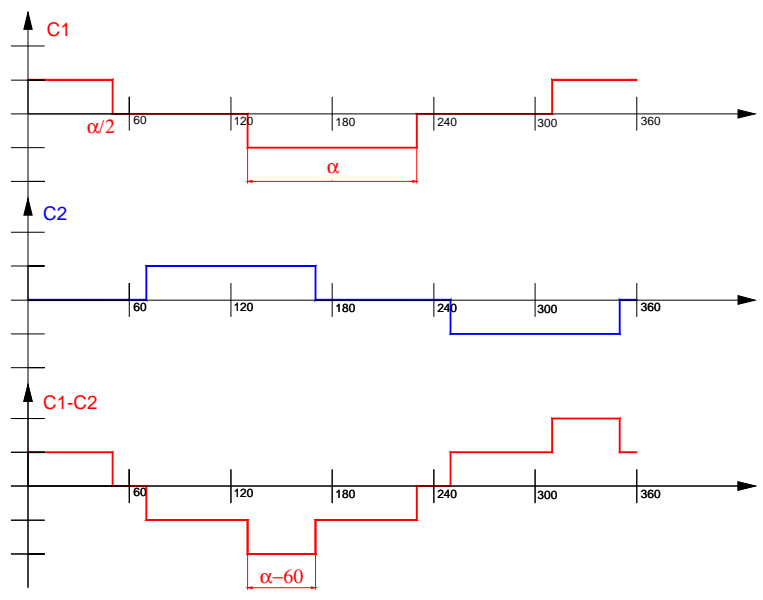

Fig. 12: Couple statique théorique lorsque la phase 1 et 2 sont alimentées en anti-série

\subsection{Détermination de la stratégie d'alimentation}

Le relevé des courbes de couple statique entre deux phases va permettre de déterminer les différentes séquences d'alimentation pour l'entraînement du rotor. Pour l'arc polaire choisi $\left(\alpha=100^{\circ}\right)$, la figure 13 montre la succession des différentes séquences lors d'une rotation complète du rotor.

Deux phases étant simultanément alimentées à tout instant, leur commande correspond à des créneaux de largeur $120^{\circ}$. Par conséquent, pour un arc polaire de $100^{\circ}$, le couple résultant théorique présente nécessairement une ondulation. Pour réduire cette ondulation, d'après la figure 12 , il faut que la largueur du plateau à couple maximal soit de $60^{\circ}$, ce qui impose un arc polaire optimal de $120^{\circ}$. 


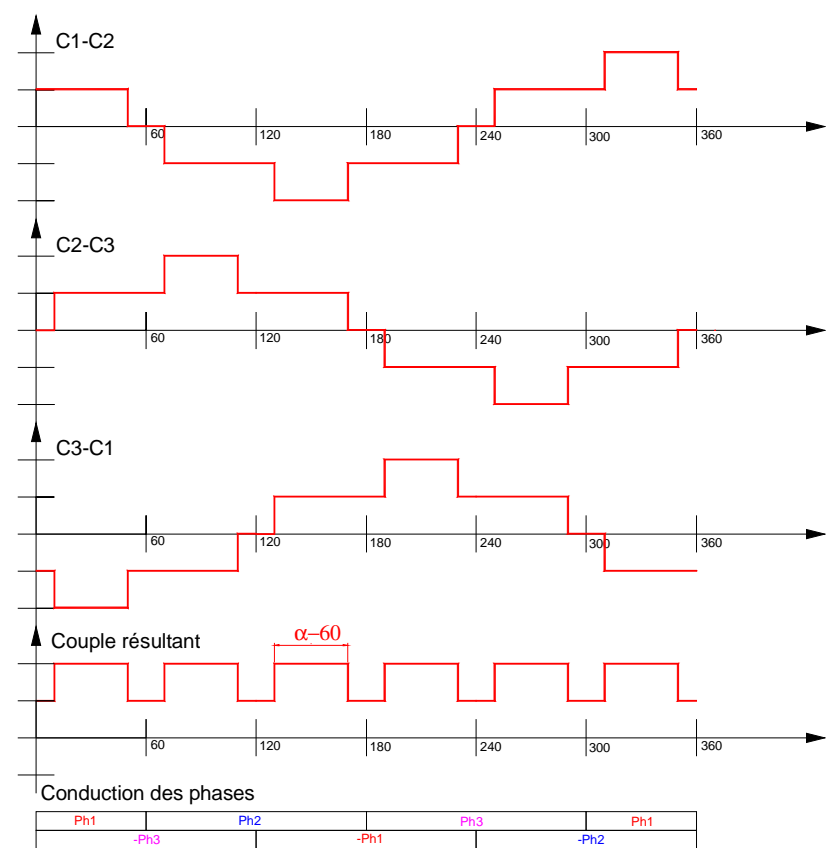

Fig. 13 : Séquences d'alimentation déduites des courbes de couple statique " entre deux phases»

Le même type de raisonnement peut être fait si on alimente une phase en série avec les deux autres. Dans ce cas, on montre que chaque phase doit être alimentée pendant $180^{\circ}$ et l'arc polaire optimal est alors de $180^{\circ}$. Ce même type de raisonnement peut être appliqué au cas plus général d'un le bobinage constitué de plusieurs encoches par pôle et par phase.

\section{COMMUTATEUR DE COURANT}

Pour alimenter le moteur à partir d'une alimentation continue stabilisée, il faut réaliser un « commutateur » de courant qui permette d'alimenter séquentiellement les phases du moteur en fonction de la position du rotor déterminée par un « capteur ».

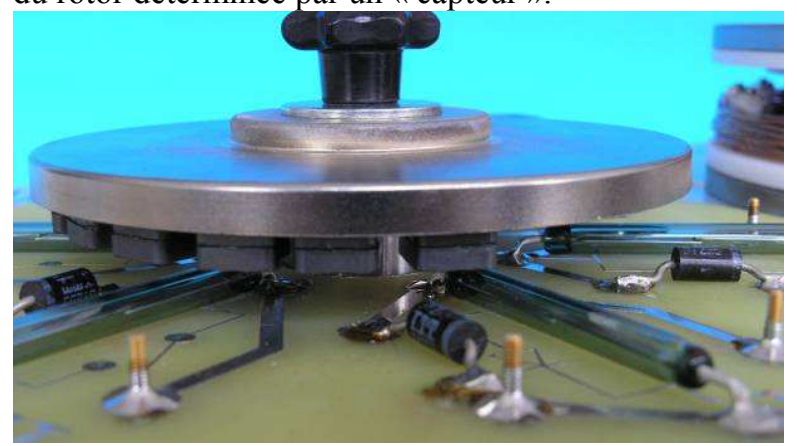

Fig. 14 : Vue du commutateur avec les REED, les aimants ferrites et les diodes de roue libre

Généralement dissociées dans les dispositifs industriels à commutation électronique [2], ces deux fonctions sont ici intégrées au sein d'un même organe, comme dans le cas des machines à courant continu ( Fig 14 ). La figure 15 donne le schéma de principe du commutateur à commande magnétique.

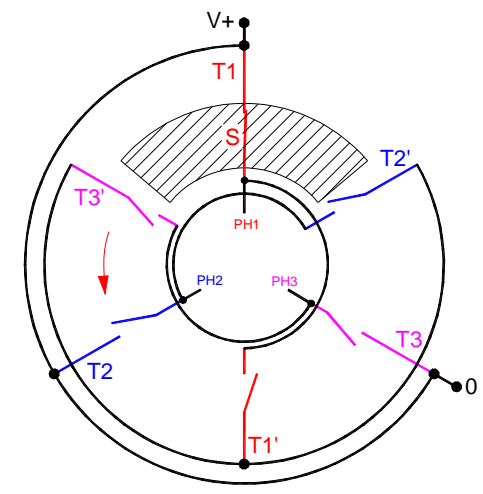

Fig. 15 : Schéma de principe du commutateur

La génération des courants en créneaux requis s'obtient en disposant les petits aimants ferrite du commutateur sur un secteur de largeur angulaire égale à $120^{\circ}$. Sur la figure 16 , on vérifie que cette configuration conduit à l'alimentation simultanée de deux phases.
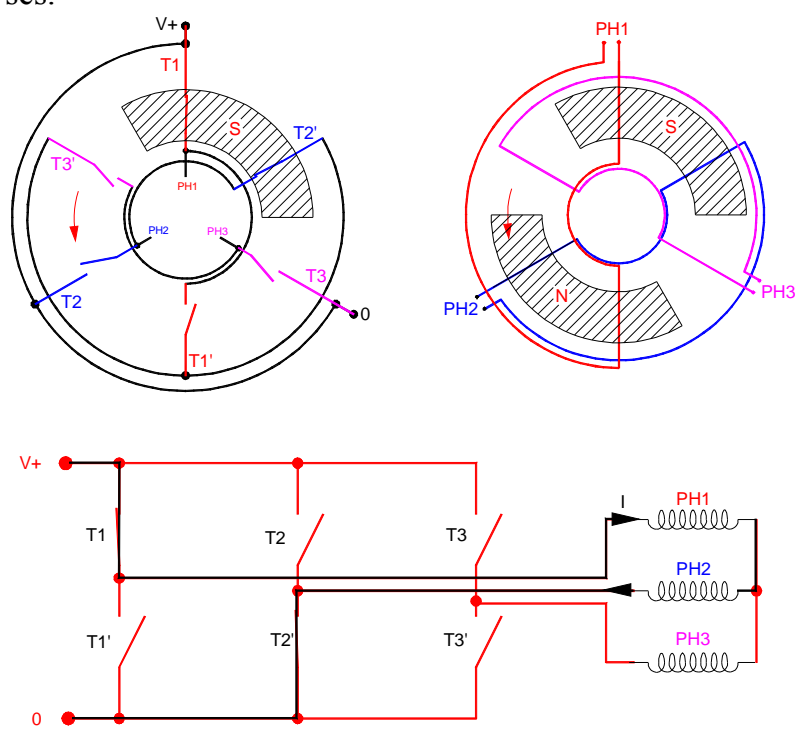

Fig. 16 : Réalisation d'une commande à $120^{\circ}$ et une séquence d'alimentation

De la même façon, si la largeur de la couronne d'aimants est de $180^{\circ}$, trois phases seront alimentées simultanément et ce pendant $180^{\circ}$ pour chaque phase. Pour éviter de court-circuiter la source continue, la largeur de la couronne doit être légèrement inférieure à $180^{\circ}$.

\section{MODE OPERATOIRE}

Les étudiants implantent leur bobinage sur le moteur, câblent le commutateur avec l'alimentation stabilisée et les phases du moteur. Pour vérifier le fonctionnement du commutateur, ils peuvent débrayer la liaison mécanique entre le moteur et le disque de commande. En mettant en marche la source de courant continu, l'entraînement manuel du disque de commande déclenche la succession des séquences d'alimentation, produisant à son tour la mise en rotation du moteur, à 
condition toutefois que le rotor reste «accroché » au champ tournant. Le fonctionnement autopiloté permettra de s'affranchir de ce défaut. A cette fin, on doit préalablement réaliser le calage du commutateur pour travailler, par exemple, à couple maximum. A cette fin, il suffit, avant d'embrayer le commutateur, d'imprimer un décalage angulaire de $\pi / 2$ à l'arbre moteur à partir de la position d'équilibre résultant de l'alimentation de deux phases en anti-série.

\section{CARACTERISATIONS, MISE EN OEU- VRE ET MESURES}

Avant la mise en route de l'autopilotage proprement dit, les élèves évaluent les propriétés de leur machine en relevant la force électromotrice. En fonction du nombre d'encoches choisi, l'allure de la fem sera plus proche d'une sinusoïde si ce nombre est important comme illustré figure 16. L'ondulation de la fem témoigne indirectement de l'ondulation de couple existant lors du fonctionnement de la machine, de même, les ondulations périodiques observées sur les deux mesures sont dues à l'espacement entre les aimants qui constituent les couronnes au rotor.

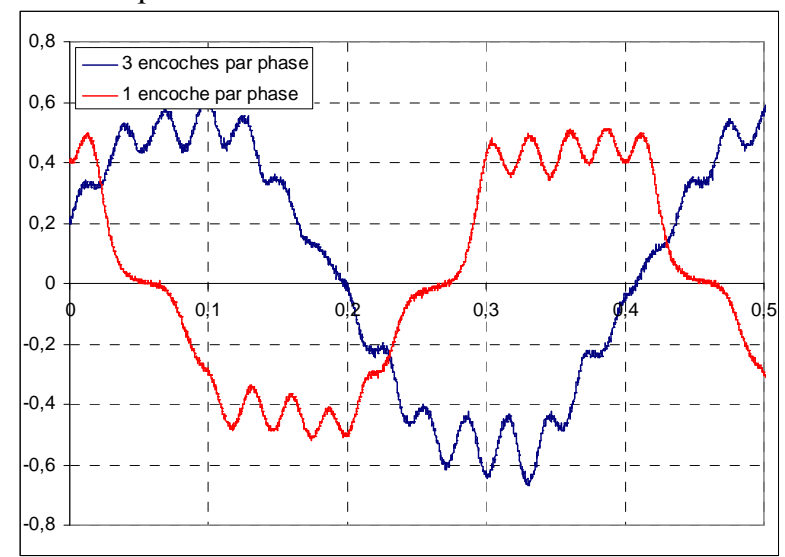

Fig. 16: Forces électromotrices obtenues pour une encoche par phase et 3 encoches par phase

Conformément à la relation (6) et (7), la mesure de la force électromotrice entre-phase permet de déduire ainsi le couple maximal fourni par la machine.

Une fois la vérification des déphasages et de l'ordonnancement des commutations faite, le moteur est actionné selon le mode opératoire détaillé précédemment. Une observation des tensions (Fig. 17) appliquées à chaque phase montre la durée des conductions de chacune d'elles, confirmant les attentes théoriques de la figure 13.

La caractérisation classique d'une machine peut alors être entreprise, qualifiant ainsi les performances en vitesse, en couple et en rendement de la machine.

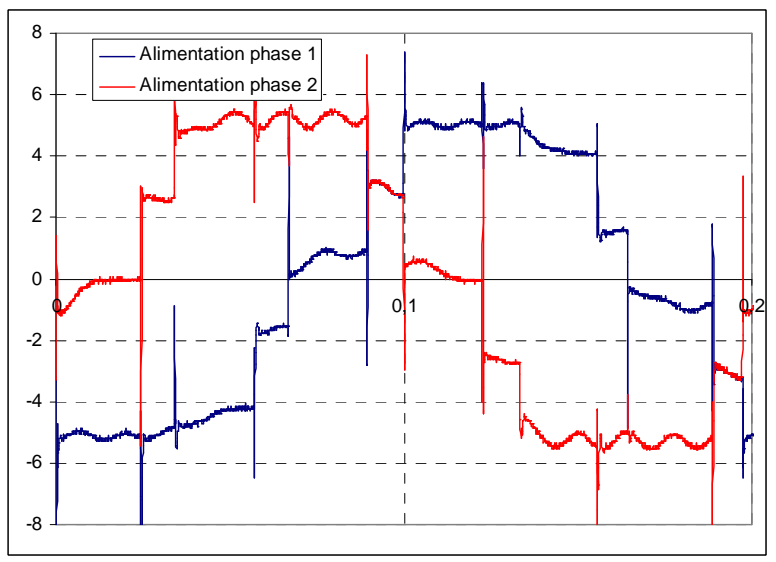

Fig. 16: Tensions d'alimentation de deux phases successives du moteur

\section{CONCLUSIONS}

La maquette MicroMAG a été développée pour faire partie de la formation des élèves ingénieurs de l'ENSEEIHT en électromagnétisme. L'approche pédagogique de ce projet qui associe les fondements théoriques et la conception pratique de la machine, remporte un vif intérêt auprès les étudiants depuis sa mise en place.

En effet, contrairement à la présentation d'une machine industrielle classique qui s'apparente généralement à une « boite noire » muni d'un rotor, le fait de passer par les étapes d'initiation et de conception permet de démystifier les principes des machines électromagnétiques. Les notions purement techniques telles que les pôles, les encoches ou l'ordonnancement des commandes prennent un sens parfaitement explicite. Il en va de même pour les propriétés des matériaux magnétiques, les forces électromotrices ou le champ magnétique.

De plus, le système d'autopilotage obtenu à partir de simples commutateurs REED permet de souligner aisément le principe des commutations sans pour autant s'enfermer dans une technologie particulière, que ce soit mécanique (collecteurs - balais) ou encore électronique. La modularité accordée par cette maquette innovante fournit un outil évolutif complet capable de s'adapter en fonction de l'accent pédagogique souhaité.

\section{BIBLIOGRAPHIE}

[1] B. Nogarede, "Electrodynamique Appliquée - Bases et principes physiques de l'électrotechnique", Dunod, 2005, ISBN 210007314 1, Paris, 260 p.

[2] J. R. Hendershot et TJE Miller, "Design of brushless permanent-magnet motors", Magna Physics Publishing and Clarendon Press, 1994, ISBN 1-88185503-01, Oxford. 